\title{
Trailblazing partnership announced for two of the largest dental firms
}

A trailblazing new partnership between Philips and \{my\}dentist has been announced making a bespoke suite of entry-level electric toothbrushes and a powered interdental flossing device accessible to more than eight million dental patients.

The collaboration between Philips, the leading technology-focussed oral healthcare innovator, and $\{\mathrm{my}\}$ dentist, Europe's largest dental care provider, will see more than 650 dental clinics in the UK and Ireland stocked with an exclusive and jointly branded portfolio of Philips oral health products that will include:

- Sonicare CleanCare Plus

- Sonicare EasyClean

- Sonicare Protective Clean 4300

- AirFloss 1.5.

These will be available in all $\{$ my $\}$ dentist clinics from November 2018, giving patients access to innovative technologies for improving their oral health.

Additionally, Zoom! take home teeth whitening will be offered in every $\{$ my\}dentist practice in the UK and Ireland from July 2019 to augment the 70 practices currently offering Zoom! chairside teeth whitening.
With a recent Oral Health Foundation survey revealing that the average spent on looking after oral health in the UK is $£ 196$ a year, this close partnership is designed to meet the needs of a population looking for entry level powered oral health products to help ensure their teeth and gums are as healthy as they can possibly be, at an affordable price.

The bespoke mydentist portfolio of oral health products includes:

- Sonicare CleanCare Plus - designed to be the perfect toothbrush for transitioning from a manual toothbrush, it removes up to three times more plaque than a manual toothbrush for a superior clean and helps improve gum health

- Sonicare EasyClean - the unique dynamic cleaning action gently and effectively reaches deep between teeth and along the gumline

- Sonicare ProtectiveClean 4300 - designed to make good oral health more accessible, it features inbuilt pressure sensors to let patients know when they are brushing too hard. The brush includes one inbuilt cleaning mode, with two intensity settings. The handle comes with an Optimal Plaque Defence brush head, charger and features BrushSync technology
- AirFloss 1.5 - designed to help inconsistent flossers develop a healthy daily interdental cleaning routine. By gently bursting away plaque that brushing missed, AirFloss Pro helps prevent cavities from forming in the spaces between teeth.

Dr Nyree Whitley, \{my\}dentist Clinical Director, said: 'Our clinicians are at the frontline of oral care and prevention and we wanted them to have the lead role in selecting the brands and products they believe in.

'Philips is a leader in oral healthcare innovation and well-respected in the industry. Working with them and our clinicians, we have been able to put together a market-leading professional range with exceptional value for money compared to high street retailers.'

Robin van Rozen, Managing Director, Personal Health, Philips UK\&I added: 'We are delighted to be working with $\{$ my\}dentist, not only to bring products and solutions to market, but more importantly to work together to improve the oral health of the nation.'

More information is available at www. philips.co.uk/dentalprofessional or by calling 08000567222.

\section{Next generation wet milling machine announced}

DGSHAPE Corporation, a wholly-owned subsidiary of Roland DG Corporation and a leading provider of digital fabrication tools, including 3D milling machines, 3D printers, and engraving machines, have announced the release of the DWX-42W the successor to the popular DWX-4W wet milling machine.

The DWX-42W can produce highlyaesthetic restorations from the latest glass ceramic and composite resins.

A host of enhancements have been made to the DWX wet platform, including new grinding burs, increased Automatic Tool Changer (ATC) capacity, ethernet connectivity, and a new, DGSHAPE engineered spindle (patent pending).

Additional key upgrades include reduced air pressure and volume requirements (increasing compatibility to working with existing compressed air supply), up to $50 \%$ faster milling times, and a reinforced frame design for increased stability and precision.

A cornerstone of all DWX dental machines, says the company, is their ease of use - operation is as simple as pressing a button.

Their open architecture offers the flexibility of using the software and scanners lab technicians are already familiar with. The six-station ATC switches out grinding burs as needed for unattended production.

Other advanced features include a Virtual Machine Panel (VPanel) which allows users to quickly configure settings for up to four machines from a single computer for highvolume production. The VPanel monitors operational status and sends email notifications at the end of milling and maintenance completion. Hisashi Bito, President of DGSHAPE, said: 'The DWX-42W represents the latest addition to our growing portfolio of dental restoration technology.
'The DWX-42W builds on the legacy of Roland dental solutions that is advanced today by DGSHAPE Corporation and affirms DGSHAPE's commitment to helping dental professionals increase their efficiency, reduce chair time for their patients, and increase their profitability.

More information is available by visiting www.rolanddg.co.uk/products/dental/ dwx-42w-wet-dental-milling-machine.

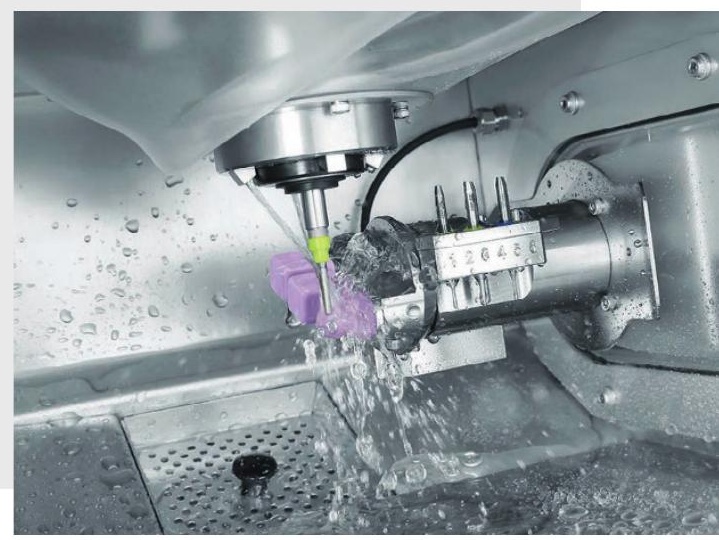

